IFUP-TH 69/96

Novenber 1996

\title{
Three-manifold invariants and their relation with the fundamental group
}

\author{
E. Guadagnini ${ }^{a, b}$ and L. Pilo ${ }^{b, c}$ \\ (a) Dipartimento di Fisica dell'Università di Pisa, \\ Piazza Torricelli, 2. 56100 Pisa. Italy \\ (b) Istituto Nazionale di Fisica Nucleare, Pisa Italy. \\ (c) Scuola Normale Superiore \\ Piazza dei Cavalieri 7. 56100 Pisa, Italy. \\ E-Mail: guadagni@ipifidpt.difi.unipi.it, pilo@ibmth.difi.unipi.it
}

\begin{abstract}
We consider the 3-manifold invariant $I(M)$ which is defined by means of the Chern-Simons quantum field theory and which coincides with the Reshetikhin-Turaev invariant. We present some arguments and numerical results supporting the conjecture that, for nonvanishing $I(M)$, the absolute value $|I(M)|$ only depends on the fundamental group $\pi_{1}(M)$ of the manifold $M$. For lens spaces, the conjecture is proved when the gauge group is $S U(2)$. In the case in which the gauge group is $S U(3)$, we present numerical computations confirming the conjecture.
\end{abstract}




\section{Introduction}

Recently, new 3-manifold invariants [1, 2] have been discovered; the algebraic aspects of their construction, which is based on the structure of simple Lie groups, are well understood [3, 4, 5, 6, 7, 8, 9]. However, the topological meaning of these invariants is still unclear. Let us denote by $I(M)$ the invariant of the 3-manifold $M$ which is closed, connected and orientable; $I(M)$ is the invariant defined by means of the Chern-Simons quantum field theory [1, 9] and coincides with the Reshetikhin-Turaev invariant [2, 3]. In general, it is not known how $I(M)$ is related, for instance, to the homotopy class of $M$ or to the fundamental group of $M$. In this article we shall formulate the following

Conjecture: for nonvanishing $I(M)$, the absolute value $|I(M)|$ only depends on the fundamental group $\pi_{1}(M)$.

In the absence of a general proof, we shall verify the validity of the conjecture for a particular class of manifolds: the lens spaces. There are examples of lens spaces $M_{1}$ and $M_{2}$ with the same fundamental group $\pi_{1}\left(M_{1}\right) \simeq \pi_{1}\left(M_{2}\right)$ which are not homeomorphic; for all these manifolds, we shall prove that (for nonvanishing invariants) $\left|I\left(M_{1}\right)\right|=\left|I\left(M_{2}\right)\right|$ when $I(M)$ is the invariant associated with the group $S U(2)$. In the case in which the gauge group is $S U(3)$, we will present numerical computations confirming the conjecture. Our results are in agreement with the computer calculations for $S U(2)$ of Freed and Gompf [10] and the expression of the $S U$ (2) invariant obtained by Jeffrey [11]. Differently from [10] and [11], our approach is based exclusively on the properties of 3-dimensional Chern-Simons quantum field theory. We shall use general surgery rules to compute $I(M)$ and, in our construction, invariance under Kirby moves is manifestly satisfied.

Our notations and conventions are described in section 2. The expression of the invariant $I(M)$ for a generic lens space is derived in section 3 and, for the gauge group $S U(2)$, the validity of our conjecture is proved in section 4 . The numerical computations for the group $S U(3)$ are reported in section 5 and the conclusions are contained in section 6 .

\section{Surgery rules}

The basic ingredient in the construction of the 3-manifold invariant $I(M)$ is a polynomial invariant $E(\mathcal{L})$ for oriented, framed and coloured links $\{\mathcal{L}\} \subset S^{3}$. In the Chern-Simons field theory, this link invariant is defined by the expectation values of the Wilson line operators [1]; each link component is framed and its colour is given by an irreducible representation of a simple compact Lie group which is called the gauge group. For example, when the gauge group is $S U(N)$ and each link component has colour corresponding to the fundamental representation of $S U(N), E(\mathcal{L})$ is determined by the skein relation [1, 12]

$$
q^{1 /(2 N)} E\left(\mathcal{L}_{+}\right)-q^{-1 /(2 N)} E\left(\mathcal{L}_{-}\right)=\left(q^{1 / 2}-q^{-1 / 2}\right) E\left(\mathcal{L}_{0}\right)
$$


where $q=\exp (-i 2 \pi / k)$ is the deformation parameter and $k$ is the renormalized coupling constant of the Chern-Simons field theory. The standard skein-related links $\mathcal{L}_{+}, \mathcal{L}_{-}$and $\mathcal{L}_{0}$ correspond to a configuration with over-crossing, under-crossing and no-crossing respectively. Moreover, under an elementary \pm 1 modification of the framing of a link component, $E(\mathcal{L})$ gets multiplied by the factor $q^{ \pm\left(N^{2}-1\right) / 2 N}$. Finally, the factorization property [1], 12] which holds for the distant union of links fixes the normalization of the unknot with preferred framing

$$
E_{0}[\text { fund. }]=\left(q^{N / 2}-q^{-N / 2}\right) /\left(q^{1 / 2}-q^{-1 / 2}\right) \quad .
$$

In general, the colour which characterizes one link component is an element of the algebra $\mathcal{T}$ which coincides with the complex extension of the representation ring of the gauge group. The sum operation in this algebra extends by linearity to $E(\mathcal{L})$; whereas the product operation in the colour algebra $\mathcal{T}$ simply corresponds to the satellites obtained from the companion links by standard cabling [6, 13]. For unitary groups, the fundamental skein relation (11), the normalization (2) of the unknot and the correspondence between cabled components and higher-dimensional representations of the gauge group uniquely determine the values of the link invariant $E(\mathcal{L})$ for arbitrary coloured link components.

Let us denote by $\mathcal{L}_{1} \# \mathcal{L}_{2}[\rho]$ the connected sum of the links $\mathcal{L}_{1}$ and $\mathcal{L}_{2}$ in which the component which connects these two links has colour given by the irreducible representation $\rho$ of the gauge group. From the properties of the Chern-Simons field theory it follows that [1, 13]

$$
E\left(\mathcal{L}_{1} \# \mathcal{L}_{2}[\rho]\right)=\frac{E\left(\mathcal{L}_{1}\right) E\left(\mathcal{L}_{2}\right)}{E_{0}[\rho]}
$$

where $E_{0}[\rho]$ is the value of the unknot with preferred framing and colour $\rho$.

For integer values of the Chern-Simons coupling constant $k(k=1,2,3, \ldots)$, the set of vanishing link invariants defines an ideal $\mathcal{I}_{k}$ of $\mathcal{T}$. Thus, for fixed integer $k$, the colour states belong to the algebra [13] of the equivalence classes

$$
\mathcal{T}_{(k)}=\mathcal{T} / \mathcal{I}_{k}
$$

Usually, $\mathcal{T}_{(k)}$ is of finite order [14 and, for appropriate values of $k, \mathcal{T}_{(k)}$ is isomorphic with the Verlinde algebra [15] which is determined by of the fusion rules of certain twodimensional conformal models [1]. We shall now concentrate on $\mathcal{T}_{(k)}$ when the gauge group $G$ is $S U(2)$ [13] or $S U(3)$ [16]. For $G=S U(2)$ and $k=1, \mathcal{T}_{(1)}$ is isomorphic with the group algebra of $Z_{2}$, which is the center of $S U(2)$. For $G=S U(2)$ and $k \geq 2$, the ideal $\mathcal{I}_{k}$ is generated by the representation with $J=(k-1) / 2$ and $\mathcal{T}_{(k)}$ is of order $(k-1)$. For $G=S U(3)$ and $k=1,2$, the algebra (41) is isomorphic with the group algebra of $Z_{3}$, which is the center of $S U(3)$. For $G=S U(3)$ and $k \geq 3$, the ideal $\mathcal{I}_{k}$ is generated by the two irreducible representations with Dynkin labels $(k-1,0)$ and $(k-2,0)$; in this case, $\mathcal{T}_{(k)}$ is of order $(k-1)(k-2) / 2$.

We shall denote by $\{\psi[i]\}$ (with $i=1,2, \ldots, \operatorname{dim}\left(\mathcal{T}_{(k)}\right)$ ) the elements of a basis in $\mathcal{T}_{(k)}$. When $G=S U(2)$ and $k \geq 2$ or when $G=S U(3)$ and $k \geq 3$, each 
$\psi[i]$ represents the equivalence class of an irreducible representation of the gauge group. For low values of $k, \psi[i]$ corresponds to an irreducible representation of the gauge group up to a nontrivial multiplicative factor [13, 16]. The unit in $\mathcal{T}_{(k)}$ will be denoted by $\psi[1] ; \psi[1]$ is the class defined by the trivial representation.

Let us now consider the definition of the 3-manifold invariant $I(M)$. Each 3manifold $M$, which is closed, connected and orientable, admits a surgery presentation [17] given by Dehn surgery on $S^{3}$. Each "honest" [17 surgery instruction can be represented by a framed link $\mathcal{L} \subset S^{3}$ with components $\left\{\mathcal{L}_{b}\right\}$ with $b=1,2, \ldots$. The surgery link $\mathcal{L}$ is not oriented and an integer surgery coefficient $r_{b}$ is attached to the component $\mathcal{L}_{b}$. The framing $\mathcal{L}_{b f}$ of $\mathcal{L}_{b}$ is specified by the linking number

$$
\ell \mathrm{k}\left(\mathcal{L}_{b}, \mathcal{L}_{b f}\right)=r_{b} .
$$

The surgery link associated to the manifold $M$ is not unique. Indeed, if the surgery links $\mathcal{L}$ and $\mathcal{L}^{\prime}$ are related by a finite sequence of Kirby moves, the corresponding manifolds are homeomorphic [18]. Therefore, each 3-manifold $M$ is characterized by a class of "equivalent" surgery links in $S^{3}$, where "equivalent" links means links related by Kirby moves.

Let $\mathcal{L} \subset S^{3}$ be a surgery link for the manifold $M$. The invariant $I(M)$ is defined in terms of the expectation value $E(\mathcal{L})$ of the Wilson line operators associated with the surgery link $\mathcal{L}$. More precisely, one introduces an (arbitrary) orientation and a particular colour state $\Psi_{0}$ for each component of $\mathcal{L}$. For fixed integer $k$, the surgery colour state $\Psi_{0} \in \mathcal{T}_{(k)}$ is [2]

$$
\Psi_{0}=a_{k} \sum_{i} E_{0}[i] \psi[i] \quad,
$$

where the sum is performed over all the elements $\{\psi[i]\}$ of the basis of $\mathcal{T}_{(k)}$. The coefficients $\left\{E_{0}[i]\right\}$ coincide with the expectation values of the unknot with preferred framing and colour $\psi[i]$. When the gauge group $G$ is $S U(2), a_{k}$ is given by [13]

$$
a_{k}=\left\{\begin{array}{cc}
1 / \sqrt{2} & k=1 \\
\sqrt{\frac{2}{k}} \sin (\pi / k) & k \geq 2 \quad ;
\end{array}\right.
$$

whereas, when $G=S U(3)$, one has [16]

$$
a_{k}=\left\{\begin{array}{cl}
1 / \sqrt{3} & k=1,2 \\
16 \cos (\pi / k) \sin ^{3}(\pi / k) /(k \sqrt{3}) & k \geq 3 .
\end{array}\right.
$$

We shall denote by $\sigma(\mathcal{L})$ the signature of the linking matrix associated with $\mathcal{L} ; \sigma(\mathcal{L})$ does not depend on the choice of the orientation of $\mathcal{L}$. Let us define the function $I(\mathcal{L})$ by means of the relation [2]

$$
I(\mathcal{L})=\exp \left[i \theta_{k} \sigma(\mathcal{L})\right] E(\mathcal{L}),
$$

where, for $G=S U(2)$, the phase factor $e^{i \theta_{k}}$ is [2, 13]

$$
e^{i \theta_{k}}=\left\{\begin{array}{cc}
\exp (-i \pi / 4) & k=1 \\
\exp [i \pi 3(k-2) /(4 k)] & k \geq 2 ;
\end{array} ;\right.
$$


and, for $G=S U(3)$, the phase factor is 16

$$
e^{i \theta_{k}}=\left\{\begin{array}{cc}
\exp (i \pi / 2) & k=1 \\
\exp (-i \pi / 2) & k=2 \\
\exp (-i 6 \pi / k) & k \geq 3
\end{array} .\right.
$$

It can be verified [2, 3, 13, 16] that $I(\mathcal{L})$ is invariant under Kirby moves and then it represents a topological invariant for the 3-manifold $M$. In what follows, we shall denote this invariant by $I(M)$.

It should be noted that the multiplicative phase factor in (9) is not a matter of convention (or choice of framing); the presence of the term $\exp \left[i \theta_{k} \sigma(\mathcal{L})\right]$ in (9) guarantees the invariance of $I(\mathcal{L})$ under Kirby moves. According to the definition (9), the normalization of the 3-manifold invariant $I(M)$ is fixed by $I\left(S^{3}\right)=1$.

In order to compare $I(M)$ with the expressions obtained in [10, 11], we need to produce the relation between the link invariants and the representation matrices of the mapping class group of the torus.

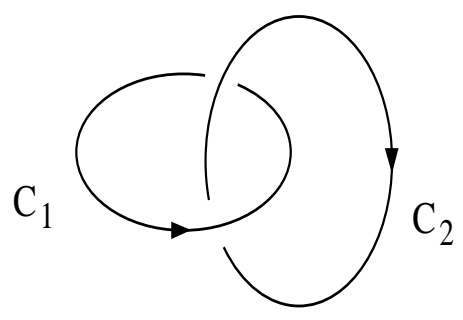

Figure 1

Let us consider the Hopf link in $S^{3}$, shown in Figure 1; let the two link components $C_{1}$ and $C_{2}$ have preferred framings and colours $\psi[i]$ and $\psi[j]$ respectively. The associated Chern-Simons expectation value is denoted by

$$
H_{i j}=E\left(C_{1}, \psi[i] ; C_{2}, \psi, \psi[j]\right) \quad .
$$

The complex numbers $\left\{H_{i j}\right\}$ where $i, j=1,2, \ldots, \operatorname{dim}\left(\mathcal{T}_{(k)}\right)$ can be understood as the matrix elements of the so-called Hopf matrix $H$. Note that $H$ is symmetric and that $E_{0}[i]=H_{1 i}=H_{i 1}$. Let $Q(i)$ be the value of the quadratic Casimir operator of the irreducible representation of the gauge group which is associated with an element of the class $\psi[i]$. One can show [14] that the matrices

$$
X_{i j}=a_{k} H_{i j} \quad ; \quad Y_{i j}=q^{Q(i)} \delta_{i j} \quad ; \quad C_{i j}=\delta_{i j^{*}} .
$$

give a projective representation of the modular group

$$
X^{2}=C \quad ;
$$




$$
(X Y)^{3}=e^{-i \theta_{k}} C
$$

This representation is isomorphic with the representation obtained in two-dimensional conformal field theories [1]; $X$ corresponds to the $S$ matrix of the conformal models and $Y$ is the analogue of the $T$ matrix.

\section{Lens Spaces}

Lens spaces, which are characterized by two integers $p$ and $r$, will be denoted by $\left\{L_{p / r}\right\}$. The fundamental group of $L_{p / r}$ is $Z_{p}$. Two lens spaces $L_{p / r}$ and $L_{p^{\prime} / r^{\prime}}$ are homeomorphic if and only if $|p|=\left|p^{\prime}\right|$ and $r= \pm r^{\prime}(\bmod p)$ or $r r^{\prime}= \pm 1(\bmod p)$. Thus, we only need [17] to consider the case in which $p>1$ and $0<r<p$; moreover, $r$ and $p$ are relatively prime. The lens spaces $L_{p / r}$ and $L_{p^{\prime} / r^{\prime}}$ are homotopic if and only if $|p|=\left|p^{\prime}\right|$ and $r r^{\prime}= \pm$ quadratic residue $(\bmod p)$. Consequently, one can find examples of lens spaces which are homotopic but are not homeomorphic; for instance, $L_{13 / 2}$ and $L_{13 / 5}$. One can also find examples of lens spaces which are not homeomorphic and are not homotopic but have the same fundamental group; for instance, $L_{13 / 2}$ and $L_{13 / 3}$.

One possible surgery instruction corresponding to the lens space $L_{p / r}$ is given the unknot [17] with rational surgery coefficient $(p / r)$. From this surgery presentation one can derive [17] a "honest" surgery presentation of $L_{p / r}$ by using a continued fraction decomposition of the ratio $(p / r)$

$$
\frac{p}{r}=z_{d}-\frac{1}{z_{d-1}-\frac{1}{\ddots \cdot-\frac{1}{z_{1}}}}
$$

where $\left\{z_{1}, z_{2}, \cdots, z_{d}\right\}$ are integers. The new surgery link $\mathcal{L}$ corresponding to a "honest" surgery presentation of $L_{p / r}$ is a chain with $d$ linked components, as shown in Figure 2, and the integers $\left\{z_{1}, z_{2}, \cdots, z_{d}\right\}$ are precisely the surgery coefficients.

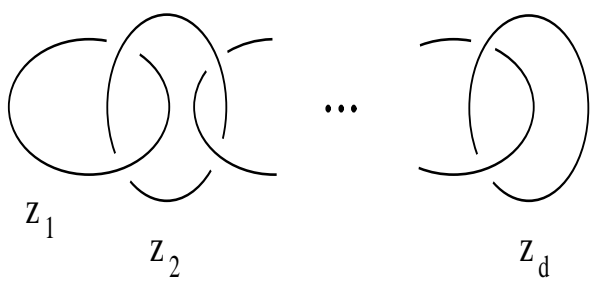

Figure 2

According to the definition (9), the lens space invariant is given by 


$$
\begin{aligned}
I\left(L_{p / r}\right)=e^{i \theta_{k} \sigma(\mathcal{L})}\left(a_{k}\right)^{d} \sum_{j_{1}, \cdots, j_{d} \in \mathcal{T}_{k}} \prod_{i=1}^{d}\left(q^{z_{i} Q\left(j_{i}\right)}\right) \times \\
\quad \times E_{0}\left[j_{1}\right] \cdots E_{0}\left[j_{d}\right] E\left(\mathcal{L} ; \psi\left[j_{1}\right], \cdots, \psi\left[j_{d}\right]\right)
\end{aligned}
$$

The link of Figure 2 can be understood as the connected sum of $(d-1)$ Hopf links $\mathcal{H}$, i.e. $\mathcal{L}=\mathcal{H} \# \mathcal{H} \cdots \# \mathcal{H}$. Therefore, by using equation (3), expression (17) can be written as

$$
I\left(L_{p / r}\right)=e^{i \theta_{k} \sigma(\mathcal{L})}\left(a_{k}\right)^{d} \sum_{j_{1}, \cdots, j_{d} \in \mathcal{T}_{k}} q\left(\sum_{i=1}^{d} z_{i} Q\left(j_{i}\right)\right) H_{1 j_{d}} H_{j_{d} j_{d-1}} \cdots H_{j_{2} j_{1}} H_{j_{1} 1}
$$

In terms of the generators (13) of the modular group, one finds

$$
I\left(L_{p / r}\right)=e^{i \theta_{k} \sigma(\mathcal{L})}\left(a_{k}\right)^{-1}[F(p / r)]_{11} \quad,
$$

where $[F(p / r)]_{11}$ is the element corresponding to the first row and the first column of the following matrix

$$
F(p / r)=X Y^{z_{d}} X Y^{z_{d-1}} X \cdots X Y^{z_{1}} X
$$

The invariant $I\left(L_{p / r}\right)$ given in equation (19) is in agreement with the expressions obtained in [10, 11] apart from an overall normalization factor.

\section{The $\mathrm{SU}(2)$ case}

In this section, we shall compute $I\left(L_{p / r}\right)$ for the gauge group $G=S U(2)$. Then, we will show that in this case our conjecture is true; i.e. when $I\left(L_{p / r}\right) \neq 0$, the absolute value $\left|I\left(L_{p / r}\right)\right|$ only depends on $p$.

For $k \geq 2$, the standard basis of $\mathcal{T}_{k}$ is $\{\psi[j]\}$; the index $j$ represents the dimension of the irreducible representation described by $\psi[j]$ and $1 \leq j \leq(k-1)$. The matrix elements of $X$ and $Y$ are

$$
\begin{aligned}
& (X)_{m n}=\frac{i}{\sqrt{2 k}}\left[\exp \left(-\frac{i \pi m n}{k}\right)-\exp \left(\frac{i \pi m n}{k}\right)\right] ; \\
& (Y)_{m n}=\xi \exp \left(-\frac{i \pi m^{2}}{2 k}\right) \delta_{m n}
\end{aligned}
$$

with

$$
\xi=\exp (i \pi / 2 k)
$$

When $k=1$, one has

$$
X=\left(\begin{array}{cc}
1 & 1 \\
1 & -1
\end{array}\right) \quad, \quad Y=\left(\begin{array}{ll}
1 & 0 \\
0 & i
\end{array}\right)
$$


The algebra $\mathcal{T}_{1}$ is isomorphic with $\mathcal{T}_{3}$ and it is easy to verify that

$$
I_{k=1}\left(L_{p / r}\right)=\left[I_{k=3}\left(L_{p / r}\right)\right]^{*} \quad .
$$

Therefore, we only need to consider the case $k \geq 2$.

In order to compute $I\left(L_{p / r}\right)$, we shall derive a recursive relation for the matrix (20); the argument that we shall use has been produced by Jeffrey [11] in a slightly different context. In fact, our final result for $I\left(L_{p / r}\right)$ is essentially in agreement with the formulae obtained by Jeffrey. Since in our approach the invariance under Kirby moves is satisfied, our derivation of $I\left(L_{p / r}\right)$ proves that the appropriate expressions given in [10, 11] really correspond to the values of a topological invariant of 3-manifolds.

Let us introduce a few definitions; with the ordered set of integers $\left\{z_{1}, z_{2}, \cdots, z_{d}\right\}$ one can define the following partial continued fraction decompositions

$$
\frac{\alpha_{t}}{\gamma_{t}}=z_{t}-\frac{1}{z_{t-1}-\frac{1}{\ddots \cdot-\frac{1}{z_{1}}}}
$$

where $1 \leq t \leq d$. The integers $\alpha_{t}$ and $\gamma_{t}$ satisfy the recursive relations

$$
\begin{aligned}
& \alpha_{m+1}=z_{m+1} \alpha_{m}-\gamma_{m}, \quad, \quad \alpha_{1}=z_{1} \quad, \quad \alpha_{0}=1 ; \\
& \gamma_{m+1}=\alpha_{m} \quad, \quad \gamma_{1}=1 \quad,
\end{aligned}
$$

and, clearly, $\alpha_{d} / \gamma_{d}=p / r$. Finally, let $F_{t}$ be the matrix

$$
F_{t}=X Y^{z_{t}} X Y^{z_{t-1}} X \cdots X Y^{z_{1}} X
$$

by definition, one has $F_{d}=F(p / r)$.

\section{Lemma 1}

The matrix element $\left(F_{t}\right)_{m n}$ is given by

$$
\begin{gathered}
\left(F_{t}\right)_{m n}=B_{t} \sum_{s\left(m, k,\left|\alpha_{t}\right|\right)}\left[e^{\frac{i \pi \gamma_{t}}{2 k \alpha_{t}}\left(s+\frac{n}{\gamma_{t}}\right)^{2}}-e^{\left.\frac{i \pi \gamma_{t}}{2 k \alpha_{t}}\left(s-\frac{n}{\gamma_{t}}\right)^{2}\right]} ;\right. \\
B_{t}=\frac{(-i)^{t+1}}{\sqrt{2 k\left|\alpha_{t}\right|}} \xi^{z_{1}+z_{2}+\cdots+z_{t}} \exp \left\{-\frac{i \pi}{4}\left[\operatorname{sign}\left(\alpha_{0} \alpha_{1}\right)+\cdots+\operatorname{sign}\left(\alpha_{t-1} \alpha_{t}\right)\right]\right\} \\
\quad \exp \left\{\frac{i \pi n^{2}}{2 k}\left[\frac{1}{\alpha_{0} \alpha_{1}}+\cdots+\frac{1}{\alpha_{t-2} \alpha_{t-1}}\right]\right\} ;
\end{gathered}
$$

where $s\left(m, k,\left|\alpha_{t}\right|\right)$ stands for the sum over a complete residue system modulo $\left(2 k\left|\alpha_{t}\right|\right)$ with the additional constraint $s \equiv m(\bmod 2 k)$.

\section{Proof}

The proof is based on induction. First of all we need to verify the validity of equations (29) and (30) when $t=1$. In this case, from the definition (28) one gets

$$
\left(F_{1}\right)_{m n}=-\frac{1}{2 k} \frac{1}{2} \xi^{z_{1}} \sum_{s=1}^{2 k} e^{-i \pi s^{2} z_{1} /(2 k)}\left[e^{-i \pi s(m+n) / k}-e^{-i \pi s(m-n) / k}+\text { c. c. }\right] \text {. }
$$


Since the sum (31) covers twice a complete residue system modulo $k$, i.e. $1 \leq s \leq 2 k$, a multiplicative factor $1 / 2$ has been introduced in (31). The change of variables $s \rightarrow-s$ shows that the last two terms in (31) are equal to the first two terms. Therefore, equation (31) can be written as

$$
\left(F_{1}\right)_{m n}=-\frac{1}{2 k} \xi^{z_{1}} \sum_{s=1}^{2 k} e^{-i \pi s^{2} z_{1} /(2 k)}\left[e^{-i \pi s(m+n) / k}-e^{-i \pi s(m-n) / k}\right] .
$$

At this point, one can use the reciprocity formula [19] reported in the appendix and one gets

$$
\begin{aligned}
\left(F_{1}\right)_{m n}= & \frac{-1}{\sqrt{2 k\left|z_{1}\right|}} \xi^{z_{1}} \exp \left\{-\frac{i \pi}{4} \operatorname{sign}\left(\alpha_{0} \alpha_{1}\right)\right\} \times \\
& \times \sum_{v=0}^{\left|z_{1}\right|-1}\left[e^{\frac{i \pi}{2 k z_{1}}(2 k v+m+n)^{2}}-e^{\frac{i \pi}{2 k z_{1}}(2 k v+m-n)^{2}}\right] .
\end{aligned}
$$

By introducing the new variable $s=2 k v+m$, one finds that in equation (33) the variable $s$ covers a complete residue system modulo $\left(2 k\left|z_{1}\right|\right)$ with the constraint that $s \equiv m(\bmod 2 k)$. Therefore, equation (33) can be written in the form

$$
\left(F_{1}\right)_{m n}=B_{1} \sum_{s\left(m, k,\left|z_{1}\right|\right)}\left[e^{\frac{i \pi}{2 k z_{1}}(s+n)^{2}}-e^{\frac{i \pi}{2 k z_{1}}(s-n)^{2}}\right] .
$$

This confirms the validity of equation (29) when $t=1$. In order to complete the proof, suppose now that (29) is true for a given $t$; we shall show that (29) is true also in the case $t+1$. Indeed, one has

$$
\left(F_{t+1}\right)_{m n}=\sum_{v=1}^{k}\left(X Y^{z_{t+1}}\right)_{m v}\left(F_{t}\right)_{v n} \quad .
$$

From equation (29) one gets

$$
\begin{aligned}
\left(F_{t+1}\right)_{m n} & =-B_{t} \frac{i \xi^{z_{t+1}}}{\sqrt{2 k}} \frac{1}{2} \sum_{v=1}^{2 k} \sum_{s\left(v, k,\left|\alpha_{t}\right|\right)} e^{-\frac{i \pi}{2 k} v^{2} z_{t+1}} \\
& {\left[e^{\frac{i \pi \gamma_{t}}{2 k \alpha_{t}}\left(s-\frac{n}{\gamma_{t}}\right)^{2}} e^{-i \pi m v / k}-e^{\frac{i \pi \gamma_{t}}{2 k \alpha_{t}}\left(s+\frac{n}{\gamma_{t}}\right)^{2}} e^{-i \pi m v / k}\right.} \\
& \left.-e^{\frac{i \pi \gamma_{t}}{2 k \alpha_{t}}\left(s-\frac{n}{\gamma_{t}}\right)^{2}} e^{i \pi m v / k}+e^{\frac{i \pi \gamma_{t}}{2 k \alpha_{t}}\left(s+\frac{n}{\gamma_{t}}\right)^{2}} e^{i \pi m v / k}\right] .
\end{aligned}
$$

Again, the last two terms can be omitted provided one introduces a multiplicative factor 2 . Moreover, because of the constraint $v=s(\bmod 2 k)$, one can set $v=s$, thus

$$
\begin{gathered}
\left(F_{t+1}\right)_{m n}=-B_{t} \frac{i \xi^{z_{t+1}}}{\sqrt{2 k}} e^{\frac{i \pi n^{2}}{2 k \alpha_{t} \gamma_{t}}} \sum_{s=0}^{2 k\left|\alpha_{t}\right|-1} \\
\left\{e^{-\frac{i \pi}{2 k \alpha_{t}}\left[\alpha_{t+1} s^{2}+2\left(\gamma_{t+1} m+n\right) s\right]}-e^{-\frac{i \pi}{2 k \alpha_{t}}\left[\alpha_{t+1} s^{2}+2\left(\gamma_{t+1} m-n\right) s\right]}\right\} .
\end{gathered}
$$


By using the reciprocity formula, one obtains the final expression for $\left(F_{t+1}\right)_{m n}$

$$
\begin{aligned}
& \left(F_{t+1}\right)_{m n}=-i B_{t} \xi^{z_{t+1}} \sqrt{\frac{\left|\alpha_{t}\right|}{\left|\alpha_{t+1}\right|}} e^{\left(\frac{i \pi n^{2}}{2 k \alpha_{t} \alpha_{t+1}}\right)} e^{\frac{-i \pi}{4} \operatorname{sign}\left(\alpha_{t} \alpha_{t+1}\right)} \\
& \sum_{v=1}^{\left|\alpha_{t+1}\right|}\left\{e^{\frac{i \pi \alpha_{t}}{2 k \alpha_{t}+1}\left(2 k v+m+\frac{n}{\alpha_{t}}\right)^{2}}-e^{\frac{i \pi \alpha_{t}}{2 k \alpha_{t}+1}\left(2 k v+m-\frac{n}{\alpha_{t}}\right)^{2}}\right\} .
\end{aligned}
$$

In terms of the variable $s=2 k v+m$, equation (38) can be rewritten in the form (29) and this concludes the proof.

From the definition (19) and Lemma 1 it follows

\section{Theorem 1}

Let $S U(2)$ be the gauge group, the 3-manifolds invariant $I_{k}\left(L_{p / r}\right)$ for $k \geq 2$ is given by

$$
\begin{gathered}
I_{k}\left(L_{p / r}\right)=\sum_{s(\bmod p)}\left\{\exp \left[\frac{i \pi(r+1)^{2}}{2 p k r}\right] \exp \left[\frac{i 2 \pi}{p}\left[r k s^{2}+(r+1) s\right]\right]\right. \\
\left.-\exp \left[\frac{i \pi(r-1)^{2}}{2 p k r}\right] \exp \left[\frac{i 2 \pi}{p}\left[r k s^{2}+(r-1) s\right]\right]\right\} \frac{e^{i \theta_{k} \sigma(\mathcal{L})} B_{d}}{a_{k}} .
\end{gathered}
$$

\section{Proof}

According to equation (19), the expression for the matrix element $[F(p / r)]_{11}$ has been written by means of a sum over a complete residue system modulo $p$.

As shown in equation (39), the expression for $I_{k}\left(L_{p / r}\right)$ is rather involved; nevertheless, $\left|I_{k}\left(L_{p / r}\right)\right|^{2}$ can be computed explicitly. Let us introduce the modulo- $p$ Croneker delta symbol defined by

$$
\delta_{p}(x)=\left\{\begin{array}{l}
0 \\
1 \quad x \equiv 0(\bmod p)
\end{array} ;\right.
$$

where $p$ and $x$ are integers. One can easily verify that, for integer $n$,

$$
\left\{\begin{aligned}
\delta_{p}(x n) & =\delta_{p}(x) \\
\delta_{p n}(x n) & =\delta_{p}(x)
\end{aligned} \quad \text { if } \quad(n, p)=1 \quad ;\right.
$$

Finally, we shall denote by $\phi(n)$ the Euler function [20] which is equal to the number of residue classes modulo $n$ which are coprime with $n$.

\section{Theorem 2}

The square of the absolute value of $I_{k}\left(L_{p / r}\right)$ is given in the following list;

for $p=2$

$$
\left|I_{k}\left(L_{2 / 1}\right)\right|^{2}=\left[1+(-1)^{k}\right] \frac{\sin ^{2}[\pi /(2 k)]}{\sin ^{2}[\pi / k]}
$$

for $p>2$ one has: 
when $p$ and $k$ are coprime integers, i.e. $(k, p)=1$,

$$
\begin{aligned}
& \left|I_{k}\left(L_{p / r}\right)\right|^{2}=\frac{1}{2}\left[1-(-1)^{p}\right] \frac{\sin ^{2}\left[\pi\left(k^{\phi(p)}-1\right) /(k p)\right]}{\sin ^{2}(\pi / k)}+ \\
& \quad \frac{1}{2}\left[1+(-1)^{p}\right]\left[1+(-1)^{p / 2}\right] \frac{\sin ^{2}\left[\pi\left(k^{\phi(p / 2)}-1\right) /(k p)\right]}{\sin ^{2}(\pi / k)} ;
\end{aligned}
$$

when the greatest common divisor of $p$ and $k$ is greater than unity, i.e. $(k, p)=$ $g>1$ and $p / g$ is odd

$$
\left|I_{k}\left(L_{p / r}\right)\right|^{2}=\frac{g}{4 \sin ^{2}(\pi / k)}\left[\delta_{g}(r-1)+\delta_{g}(r+1)\right] \quad ;
$$

when $(k, p)=g>1$ and $p / g$ is even

$$
\begin{aligned}
& \left|I_{k}\left(L_{p / r}\right)\right|^{2}=\frac{g}{4 \sin ^{2}(\pi / k)}\left\{\delta_{g}(r+1)\left[1+(-1)^{k p / 2 g^{2}}(-1)^{(r+1) / g}\right]+\right. \\
& \left.\delta_{g}(r-1)\left[1+(-1)^{k p / 2 g^{2}}(-1)^{(r-1) / g}\right]\right\}
\end{aligned}
$$

\section{Proof}

From Theorem 1 it follows that the square of the absolute value of the lens space invariant is

$$
\left|I_{k}\left(L_{p / r}\right)\right|^{2}=a(k)^{-2}(2 k p)^{-1} \mathcal{S}(k, p, r) \quad,
$$

with

$$
\begin{aligned}
& \mathcal{S}(k, p, r)=\sum_{s, t(\bmod p)}\left\{\exp \left\{\frac{i 2 \pi}{p}\left[k r\left(s^{2}-t^{2}\right)+(r+1)(s-t)\right]\right\}\right. \\
&- \exp \left(\frac{i 2 \pi}{k p}\right) \exp \left\{\frac{i 2 \pi}{p}\left[k r\left(s^{2}-t^{2}\right)+r(s-t)+s+t\right]\right\} \\
&-\exp \left(-\frac{i 2 \pi}{k p}\right) \exp \left\{\frac{i 2 \pi}{p}\left[k r\left(s^{2}-t^{2}\right)+r(s-t)-s-t\right]\right\} \\
&\left.+\exp \left\{\frac{i 2 \pi}{p}\left[k r\left(s^{2}-t^{2}\right)+(r-1)(s-t)\right]\right\}\right\}
\end{aligned}
$$

The indices $s$ and $t$ run over a complete residue system modulo $p$. When $p=2$, each sum contains only two terms and the evaluation of (47) is straightforward; the corresponding result is shown in equation (42). Let us now consider the case in which $p>2$. By means of the change of variables $s \rightarrow s+t$, the sum in $t$ becomes a geometric sum and one obtains

$$
\mathcal{S}(k, p, r)=p \sum_{s(\bmod p)}\left\{\exp \left\{\frac{i 2 \pi}{p}\left[k r s^{2}+(r+1) s\right]\right\} \delta_{p}(2 k r s)\right.
$$




$$
\begin{aligned}
& -\exp \left(\frac{i 2 \pi}{k p}\right) \exp \left\{\frac{i 2 \pi}{p}\left[k r s^{2}+(r+1) s\right]\right\} \delta_{p}(2 k r s+2) \\
& -\exp \left(\frac{-i 2 \pi}{k p}\right) \exp \left\{\frac{i 2 \pi}{p}\left[k r s^{2}+(r-1) s\right]\right\} \delta_{p}(2 k r s-2) \\
& \left.+\exp \left\{\frac{i 2 \pi}{p}\left[k r s^{2}+(r-1) s\right]\right\} \delta_{p}(2 k r s)\right\}
\end{aligned}
$$

By using properties (41), one can determine the values of $s$ which give contribution to (48). Let us start with $(k, p)=1$. Clearly, in this case one has

$$
\delta_{p}(2 r k s) \neq 0 \Rightarrow\left\{\begin{array}{cc}
s=p & p \text { odd } \\
s=p, p / 2 & p \text { even }
\end{array}\right.
$$

When $(k, p)=1$ and $p$ is odd, one gets

$$
\delta_{p}(2 k r s \mp 2)=\delta_{p}(k r s \mp 1) .
$$

The delta gives a non-vanishing contribution if and only if the following congruence is satisfied

$$
r k s= \pm 1(\bmod p)
$$

The unique solution 20 to (51) is given by

$$
s= \pm(r k)^{\phi(p)-1} .
$$

When $(k, p)=1$ and $p$ is even, one finds two solutions

$$
s_{1}= \pm(r k)^{\phi(p / 2)-1} \quad, \quad s_{2}= \pm(r k)^{\phi(p / 2)-1}+p / 2 \quad .
$$

Let us now examine the case $(p, k)=g>1$. We introduce the integer $\beta$ defined by $p=g \beta$. For $\beta$ odd, one has

$$
\delta_{p}(2 k r s)=\delta_{\beta}(s)
$$

Within the residues of a complete system modulo $p$, the values of $s$ giving nonvanishing contribution are of the form $s=\alpha \beta$ with $1 \leq \alpha \leq g$. When $\beta$ is even, one gets

$$
\delta_{p}(2 k r s)=\delta_{\beta}(2 s)=\delta_{\beta / 2}(s) \quad .
$$

The solutions of the associated congruence are

$$
s=\alpha \frac{\beta}{2} \quad 1 \leq \alpha \leq 2 g \quad .
$$

When $(k, p)=g>1$ and $p$ is odd, $\delta_{p}[2 r(k s \pm 1)]$ does not contribute because $r k s= \pm 1(\bmod p)$ has no solutions. On the other hand, if $p$ is even we have

$$
\delta_{p}[(2 r k s \pm 2)]=\delta_{p / 2}(r k s \pm 1)
$$


The delta function (57) is non-vanishing when $(p / 2, k)=1$ and, in this case, the two solutions are $s_{1}= \pm(r k)^{\phi(p / 2)-1}$ and $s_{2}=s_{1}+p / 2$. This exhausts the analysis of the modulo $p$ Croneker deltas when $p>2$.

At this stage, Theorem 2 simply follows from the substitution of the values of $s$ for which the various Croneker deltas modulo $p$ are non vanishing. In the case $(k, p)=1$ and $p$ odd, the algebraic manipulations are straightforward. When $(k, p)=1$ and $p$ even, the evaluation of (48) needs some care. In this case, one has to deal with factors of the form

$$
\exp \left[\frac{i \pi}{b}\left(a^{\phi(b)}-1\right)\right] \quad ;
$$

with $b>2$ even and $(a, b)=1$. In appendix $\mathrm{B}$, it is shown that terms of the type (58) are trivial because actually

$$
a^{\phi(b)} \equiv 1 \quad(\bmod 2 b)
$$

Finally, the derivation of equations (44) and (45) is straightforward.

Let us now consider the dependence of $\left|I\left(L_{p / r}\right)\right|^{2}$ on $r$. As shown in equations (44) and (45)), $\left|I\left(L_{p / r}\right)\right|^{2}$ depends on $r$. However, this dependence is rather peculiar: when $I\left(L_{p / r}\right) \neq 0,\left|I\left(L_{p / r}\right)\right|^{2}$ does not depend on $r$. Indeed, when expression (44) is different from zero, its values are given by

$$
0 \neq(44)=\left\{\begin{array}{cc}
\sin ^{-2}(\pi / k) & \text { for } g=2 ; \\
(g / 4) \sin ^{-2}(\pi / k) & \text { for } g>2 .
\end{array}\right.
$$

Similarly, when expression (45) is different from zero, its value is given by

$$
0 \neq(45)=\frac{g}{2 \sin ^{2}(\pi / k)} .
$$

To sum up, when $I\left(L_{p / r}\right) \neq 0,\left|I\left(L_{p / r}\right)\right|^{2}$ only depends on $p$ and, therefore, it is a function of the fundamental group $\pi_{1}\left(L_{p / r}\right)=Z_{p}$. Thus, Theorem 2 proves the validity of our conjecture for the lens spaces when the gauge group is $S U(2)$.

\section{The $\mathrm{SU}(3)$ case}

In this section we shall present numerical computations confirming the validity of our conjecture for lens spaces when the gauge group is $S U(3)$. As in the $S U(2)$ case, the $S U(3)$ Chern-Simons field theory can be solved explicitly in any closed, connected and orientable three-manifold [16]. The general surgery rules for $S U(3)$ and for any integer $k$ have been derived in [16]. In particular, it turns out that

$$
I_{k=1}\left(L_{p / r}\right)=\left[I_{k=2}\left(L_{p / r}\right)\right]^{*}=I_{k=4}\left(L_{p / r}\right) .
$$

Therefore, we only need to consider the case $k \geq 3$. For $k \geq 3$, the matrices which give a projective representation of the modular group have the following form

$$
X_{(m, n)(a, b)}=\frac{i}{k \sqrt{3}} q^{-2} q^{-[(m+n)(a+b+3)+(m+3) b+(n+3) a] / 3}
$$




$$
\begin{gathered}
{\left[1+q^{(n+1)(a+b+2)+(m+1)(b+1)}+q^{(m+1)(a+b+2)+(n+1)(a+1)}\right.} \\
\left.-q^{(m+1)(b+1)}-q^{(n+1)(a+1)}-q^{(m+n+2)(a+b+2)}\right] ; \\
Y_{(a, b)(m, n)}=q^{\left[m^{2}+n^{2}+m n+3(m+n)\right] / 3} \delta_{a m} \delta_{b n} ; \\
C_{(a, b)(m, n)}=\delta_{a n} \delta_{b m} \quad ;
\end{gathered}
$$

where each irreducible representation of $S U(3)$ has been denoted by a couple of nonnegative integers $(m, n)$ (Dynkin labels).

By using equation (18), we have computed $I_{k}\left(L_{p / r}\right)$ numerically for some examples of lens spaces. In particular, we have worked out the value of the invariant for the lens spaces $L_{p / r}$, with $p \leq 20$ and $3 \leq k \leq 50$. In all these cases, the results are in agreement with our conjecture.

Our calculations have been performed on a Pentium based PC running Linux. For instance, the results of the computations for the cases $L_{8 / 1}, L_{8 / 3}, L_{15 / 1}, L_{15 / 2}, L_{15 / 4}$ with $3 \leq k \leq 50$ are shown in Tables $1,2,3,4,5$. The spaces $L_{8 / 1}$ and $L_{8 / 3}$ are not homotopically equivalent; as shown in Tables 1 and 2, the phase of the invariant distinguishes these two manifolds. The case in which $p=15$ is more interesting because there are two different spaces belonging to the same homotopy class; $L_{15 / 1}$ and $L_{15 / 4}$ are homotopically equivalent and $L_{15 / 2}$ represents the other homotopy class. The phase of the invariant distinguishes the manifolds of the same homotopy class.

\section{Conclusions}

In this article, we have presented some arguments and numerical results supporting the conjecture that, for nonvanishing $I(M)$, the absolute value $|I(M)|$ only depends on the fundamental group $\pi_{1}(M)$. Since the Turaev-Viro invariant [21] coincides [3] with $|I(M)|^{2}$, our conjecture gives some hints on the topological interpretation of the Turaev-Viro invariant. For the gauge group $S U(2),|I(M)|^{2}$ can be understood as the improved partition function of the Euclidean version of $(2+1)$ gravity with positive cosmological constant [22, 23]. In this case, our conjecture suggests that, for instance, the semiclassical limit is uniquely determined by the fundamental group of the universe.

Finally, one may ask for which values of $k$ the equality $I_{k}(M)=0$ is satisfied and what the meaning of this fact is. The complete solution to this problem is not known. From the field theory point of view, gauge invariance of the factor $\exp \left(i S_{C S}\right)$ (where $S_{S C}$ is the Chern-Simons action) in the functional measure gives nontrivial constraints on the admissible values of $k$ in a given manifold $M$. In certain cases [9] one finds that, in correspondence with the "forbidden" values of $k$, the invariant $I_{k}(M)$ vanishes. So, it is natural to expect that $I_{k}(M)=0$ is related to a breaking of gauge invariance for large gauge transformations. From the mathematical point of view, $I_{k}(M)=0$ signals the absence of the natural extension of $E(\mathcal{L})$ to an invariant $E_{M}(\mathcal{L})$ of links in the manifold $M$. More precisely, when $I_{k}(M) \neq 0$ for fixed integer $k$, one can define [13] an invariant $E_{M}(\mathcal{L})$ of oriented, framed and 
coloured links $\{\mathcal{L} \subset M\}$ with the following property: if the link $\mathcal{L}$ belongs to a three-ball embedded in $M$, then one has $E_{M}(\mathcal{L})=E(\mathcal{L})$. The values of the invariant $E_{M}(\mathcal{L})$ correspond to the vacuum expectation values of the Wilson line operators associated with links in the manifold $M$. When $I_{k}(M)=0$, the invariant $E_{M}(\mathcal{L})$ cannot be constructed; consequently, for these particular values of $k$, the quantum Chern-Simons field theory is not well defined in $M$.

Acknowledgments. We wish to thank Turaev for useful discussions.

\section{Appendix A}

The generalized Gauss sums have a very useful property which can be expressed by means of the so-called reciprocity formula [19]

$$
\sum_{n=0}^{|c|-1} e^{i \frac{\pi}{c}\left(a n^{2}+b n\right)}=\sqrt{\left|\frac{c}{a}\right|} e^{i \frac{\pi}{4 a c}\left(|a c|-b^{2}\right)} \sum_{n=0}^{|a|-1} e^{-i \frac{\pi}{a}\left(c n^{2}+b n\right)},
$$

where the integers $a, b, c$ satisfy the relations

$$
a c \neq 0 \quad, \quad a c+b \text { is even } .
$$

\section{Appendix B}

Lemma 2 Let $a, b$ two integers, with $(a, b)=1$ and $b>2$ even; one has

$$
a^{\phi(b)} \equiv 1 \quad(\bmod 2 b)
$$

\section{Proof}

The proof consists of two parts: firstly, it is shown by induction that Lemma 2 holds when $b=2^{m}$ with $m>1$ integer. Secondly, equation (68) is proved when $b=2^{m} c$ with $m \geq 1$ and $c$ odd integer.

Since $b$ is even, $a$ is clearly odd and can be written in the form $a=(2 f+1)$. When $b$ is of the type $b=2^{m}$, the condition $b>2$ implies that $m \geq 2$. Let us now consider the case $m=2$; one has $\phi(b)=\phi\left(2^{2}\right)=2$, therefore

$$
a^{\phi(b)}=(2 f+1)^{2}=1+4 f(f+1) \equiv 1 \quad\left(\bmod 2^{3}\right) .
$$

Thus, Lemma 2 is satisfied when $b=2^{2}$. Suppose now that equation (68) holds when $b=2^{n}$ for a certain $n$. We need to prove that $(68)$ is true also for $b=2^{(n+1)}$. Indeed, $\phi\left(2^{n+1}\right)=2^{n}$ and one gets

$$
(2 f+1)^{\phi\left(2^{n+1}\right)}=\left[(2 f+1)^{\phi\left(2^{n}\right)}\right]^{2} \quad .
$$


By using the induction hypothesis

$$
(2 f+1)^{\phi\left(2^{n}\right)}=1+N 2^{n+1},
$$

one finds

$$
\left[(2 f+1)^{\phi\left(2^{n}\right)}\right]^{2}=1+2^{n+2} N\left(1+2^{n} N\right) \equiv 1 \quad\left(\bmod 2^{n+2}\right) .
$$

Therefore, equation (68) is also satisfied when $b=2^{(n+1)}$. To sum up, for $m>1$ and $a$ odd, one has

$$
a^{\phi\left(2^{m}\right)} \equiv 1 \quad\left(\bmod 2^{m+1}\right)
$$

Let us now consider the general case in which $b=2^{m} c$ with $c$ odd integer. From Euler Theorem [20] it follows that

$$
a^{\phi(b)} \equiv 1 \quad(\bmod b) \quad \Rightarrow \quad a^{\phi(b)} \equiv 1 \quad(\bmod c)
$$

On the other hand, $\phi\left(2^{m} c\right)=\phi\left(2^{m}\right) \phi(c)$ and, for $m>1$, equation (73) implies

$$
a^{\phi(b)}=a^{\phi(c) \phi\left(2^{m}\right)} \equiv 1 \quad\left(\bmod 2^{m+1}\right) \quad .
$$

Since $\left(2^{m+1}, c\right)=1$, from equations $(74)$ and (75) one gets

$$
a^{\phi(b)} \equiv 1 \quad\left(\bmod 2^{m+1} c\right) \equiv 1 \quad(\bmod 2 b)
$$

Finally, we need to consider the case $b=2 c$. Since $\phi(c)$ is even, one gets

$$
a^{\phi(2 c)}=[1+4 f(f+1)]^{\phi(c) / 2} \equiv 1 \quad\left(\bmod 2^{2}\right)
$$

Equations (73) and (77) imply

$$
a^{\phi(2 c)} \equiv 1 \quad\left(\bmod 2^{2} c\right)
$$

This concludes the proof.

\section{References}

[1] E. Witten, Commun. Math. Phys. 121 (1989) 351-399.

[2] N.Y. Reshetikhin, V.G. Turaev, Commun. Math. Phys. 127 (1990) 1-26 and Invent. Math. 103 (1991) 547-597.

[3] V.G. Turaev, Quantum invariants of knots and three manifolds, de Gruyter Studies in Mathematics 18 (Berlin, 1994).

[4] R. Kirby and P. Melvin, Invent. Math. 105 (1991) 473-545.

[5] W.B.R. Lickorish, Pacific J. Math. 149 (1991) 337-347. 
[6] H.R. Morton, P.M. Strikland, Satellites and Surgery Invariants, in Knots 90 ed. Hawauchi; de Gruyter, (Berlin, 1992).

[7] L.H. Kauffman and L.L. Sostenes Temperley-Lieb recoupling theory and invariants of 3-manifolds, Princeton University Press (Princeton, 1994).

[8] T. Kohno, Topology 31 (1992) 203-230.

[9] E. Guadagnini and S. Panicucci, Nucl. Phys. B 388 (1992) 159.

[10] D.S. Freed and R.E. Gompf, Commun. Math. Phys. 14X (1991) 79-117.

[11] L. C. Jeffrey, Commun. Math Phys. 147 (1992) 563-604.

[12] E. Guadagnini, Int. Journ. Mod. Phys. A7 (1992) 877.

[13] E. Guadagnini, The Link Invariants of the Chern-Simons Field Theory, de Gruyter Expositions in Mathematics, Walter de Gruyter (Berlin, 1993).

[14] E. Guadagnini and L. Pilo, Nucl. Phys. B 433 (1995) 597.

[15] E. Verlinde, Nucl. Phys. B 300 (1988) 360.

[16] E. Guadagnini and L. Pilo, Jour. Geom. Phys. 14 (1994) 236; and Jour. Geom. Phys. 14 (1994) 365.

[17] D. Rolfsen, Knots and Links, (Publish or Perish, 1976).

[18] R. Kirby, Invent. Math. 45 (1978) 35-56; R. Fenn and C. Rourke, Topology 18 (1979) 1-15; D. Rolfsen, Pacific Journ. Math. 110 (1984) 377-386.

[19] C.L. Siegel, Über das quadratische Reziprozitätsgesetz algebraischen Zahlkörpern, Nachr. Acad. Wiss. Göttingen Math. Phys. Kl., 1 (1960) 1-16.

[20] Hua Loo-Keng, Introduction to number theory, Springer-Verlag (New York, 1982).

[21] V.G. Turaev and O.Y. Viro, Topology 31 (1992) 865.

[22] F. Archer and R. Williams, Phys. Lett. B 273 (1991) 438.

[23] E. Guadagnini and P. Tomassini, Phys. Lett. B 336 (1994) 330. 


\begin{tabular}{|c|c|c|}
\hline • & $L_{8 / 1}$ & • \\
\hline$k$ & $I_{k}$ & $\left|I_{k}\right|$ \\
\hline 3 & 1.000000000 - i 0.000000175 & 1.000000000 \\
\hline 4 & $-1.000000000+$ i 0.000000012 & 1.000000000 \\
\hline 5 & $-0.499999839+$ i 1.538841821 & 1.618033989 \\
\hline 6 & $-2.000000000+$ i 0.000000175 & 2.000000000 \\
\hline 7 & -1.000000000 - i 0.000000095 & 1.000000000 \\
\hline 8 & $-6.828427084+$ i 6.828427165 & 9.656854249 \\
\hline 9 & $-0.499999950+$ i 0.866025433 & 1.000000000 \\
\hline 10 & $-4.236067816+$ i 3.077683759 & 5.236067977 \\
\hline 11 & -2.073846587 - i 14.423920506 & 14.572244935 \\
\hline 12 & -7.464102180 - i 12.928202904 & 14.928203230 \\
\hline 13 & -12.373524802 - i 17.926145664 & 21.781891892 \\
\hline 14 & $18.195669358+$ i 0.000000868 & 18.195669358 \\
\hline 15 & $5.657005398+$ i 4.110054701 & 6.992443043 \\
\hline 16 & $63.431390926+$ i 26.274142180 & 68.657642707 \\
\hline 17 & $6.721172941+$ i 2.603796085 & 7.207906752 \\
\hline 18 & $15.581719525+$ i 26.988328071 & 31.163437478 \\
\hline 19 & -69.185356387 - i 11.544994773 & 70.142001987 \\
\hline 20 & $-66.118464248+$ i 0.000001734 & 66.118464248 \\
\hline 21 & $-90.016155013+$ i 0.000000715 & 90.016155013 \\
\hline 22 & 43.367008373 - i 50.048195295 & 66.223253224 \\
\hline 23 & 22.973052202 - i 3.157573698 & 23.189036183 \\
\hline 24 & 219.054514271 - i 58.695485329 & 226.781922164 \\
\hline 25 & 23.000602198 - i 5.905551129 & 23.746646829 \\
\hline 26 & $84.434763344+$ i 44.314782640 & 95.357376335 \\
\hline 27 & $-185.409752744+$ i 67.483626877 & 197.308936212 \\
\hline 28 & $-161.491033989+\mathrm{i} 77.769978391$ & 179.241523084 \\
\hline 29 & $-214.342425435+$ i 99.165358161 & 236.170369862 \\
\hline 30 & 50.544948881 - i 155.561366312 & 163.566899299 \\
\hline 31 & 47.834849646 - i 26.550506056 & 54.709251617 \\
\hline 32 & 443.615385766 - i 296.414325177 & 533.531688523 \\
\hline 33 & 46.894822945 - i 30.137471278 & 55.743982582 \\
\hline 34 & $211.662329441+$ i 39.566544008 & 215.328709440 \\
\hline 35 & $-344.610722364+$ i 250.374335362 & 425.962272715 \\
\hline 36 & $-290.376668930+$ i 243.654963011 & 379.059824907 \\
\hline 37 & $-381.345093602+$ i 307.914657384 & 490.138262785 \\
\hline 38 & 27.064369107 - i 326.618342223 & 327.737732877 \\
\hline 39 & 79.852315000 - i 70.742976321 & 106.681586554 \\
\hline 40 & 734.287201006 - i 734.287220264 & 1038.438931957 \\
\hline 41 & 78.053570702 - i 75.119028091 & 108.329258655 \\
\hline 42 & 408.590935390 - i 0.000001624 & 408.590935390 \\
\hline 43 & $-545.541472393+$ i 565.843251992 & 785.998781122 \\
\hline 44 & $-452.077098514+$ i 521.724781996 & 690.340822456 \\
\hline 45 & $-590.050989168+$ i 655.318028318 & 881.817377951 \\
\hline 46 & -39.324647251 - i 574.905929557 & 576.249299975 \\
\hline 47 & 118.947578576 - i 140.691854636 & 184.235513433 \\
\hline 48 & 1090.316030520 - i 1420.927547540 & 1791.039960963 \\
\hline 49 & 116.363242798 - i 145.914887153 & 186.632147733 \\
\hline 50 & 687.196328608 - i 86.813088226 & 692.658145364 \\
\hline
\end{tabular}




\begin{tabular}{|c|c|c|}
\hline$\bullet$ & $L_{8 / 3}$ & $\bullet$ \\
\hline$k$ & $I_{k}$ & $\left|I_{k}\right|$ \\
\hline 3 & $1.000000000+$ i 0.000000000 & 1.000000000 \\
\hline 4 & $-1.000000000+$ i 0.000000000 & 1.000000000 \\
\hline 5 & 1.309016994 - i 0.951056516 & 1.618033989 \\
\hline 6 & $-2.000000000+$ i 0.000000000 & 2.000000000 \\
\hline 7 & $-0.623489802+$ i 0.781831482 & 1.000000000 \\
\hline 8 & $0.000000000+$ i 0.000000000 & 0.000000000 \\
\hline 9 & $-0.500000000+$ i 0.866025404 & 1.000000000 \\
\hline 10 & $1.618033989+$ i 4.979796570 & 5.236067977 \\
\hline 11 & 6.053529319 - i 13.255380237 & 14.572244935 \\
\hline 12 & $-7.464101615+$ i 12.928203230 & 14.928203230 \\
\hline 13 & 7.723965314 - i 20.366422715 & 21.781891892 \\
\hline 14 & $-16.393731622+$ i 7.894805057 & 18.195669358 \\
\hline 15 & $-2.160783733+$ i 6.650208521 & 6.992443043 \\
\hline 16 & $0.000000000+$ i 0.000000000 & 0.000000000 \\
\hline 17 & $-1.972537314+$ i 6.932749548 & 7.207906752 \\
\hline 18 & $15.581718739+$ i 26.988328525 & 31.163437478 \\
\hline 19 & 17.218843527 - i 67.995675380 & 70.142001987 \\
\hline 20 & $-20.431729095+$ i 62.882396270 & 66.118464248 \\
\hline 21 & 20.030478885 - i 87.759262069 & 90.016155013 \\
\hline 22 & $-55.710545730+$ i 35.802993757 & 66.223253224 \\
\hline 23 & $-4.717948848+$ i 22.704016336 & 23.189036183 \\
\hline 24 & $0.000000000+$ i 0.000000000 & 0.000000000 \\
\hline 25 & $-4.449677900+$ i 23.326028428 & 23.746646829 \\
\hline 26 & $54.169163837+$ i 78.477582217 & 95.357376335 \\
\hline 27 & 34.262337211 - i 194.311370120 & 197.308936212 \\
\hline 28 & $-39.884991120+$ i 174.747563877 & 179.241523084 \\
\hline 29 & 38.208113963 - i 233.059184818 & 236.170369862 \\
\hline 30 & $-132.328401250+$ i 96.142211171 & 163.566899299 \\
\hline 31 & $-8.284500381+$ i 54.078362271 & 54.709251617 \\
\hline 32 & $0.000000000+$ i 0.000000000 & 0.000000000 \\
\hline 33 & $-7.933195866+$ i 55.176589215 & 55.743982582 \\
\hline 34 & $129.764538515+$ i 171.836019661 & 215.328709440 \\
\hline 35 & 57.178306982 - i 422.107212669 & 425.962272715 \\
\hline 36 & $-65.823047822+\mathrm{i} 373.301054424$ & 379.059824907 \\
\hline 37 & 62.256293514 - i 486.168356194 & 490.138262785 \\
\hline 38 & $-258.631121471+$ i 201.300681961 & 327.737732877 \\
\hline 39 & $-12.859044288+$ i 105.903757675 & 106.681586554 \\
\hline 40 & $0.000000000+$ i 0.000000000 & 0.000000000 \\
\hline 41 & $-12.423570453+$ i 107.614511930 & 108.329258655 \\
\hline 42 & $254.752281347+$ i 319.449256739 & 408.590935390 \\
\hline 43 & 85.965636475 - i 781.283554973 & 785.998781122 \\
\hline 44 & $-98.245742501+$ i 683.314148273 & 690.340822456 \\
\hline 45 & 92.175015400 - i 876.986690089 & 881.817377951 \\
\hline 46 & $-447.003088251+$ i 363.663986140 & 576.249299975 \\
\hline 47 & $-18.441181139+$ i 183.310248618 & 184.235513433 \\
\hline 48 & $0.000000000+$ i 0.000000000 & 0.000000000 \\
\hline 49 & $-17.920983557+$ i 185.769741658 & 186.632147733 \\
\hline 50 & $441.516918550+$ i 533.702273720 & 692.658145364 \\
\hline
\end{tabular}




\begin{tabular}{|c|c|c|}
\hline$\bullet$ & $L_{15 / 1}$ & $\bullet$ \\
\hline$k$ & $I_{k}$ & $\left|I_{k}\right|$ \\
\hline 3 & 1.000000000 - i 0.000000175 & 1.000000000 \\
\hline 4 & $0.000000021+$ i 1.732050808 & 1.732050808 \\
\hline 5 & $-2.665351925+$ i 1.936491953 & 3.294556414 \\
\hline 6 & $3.000000303+$ i 3.464101353 & 4.582575695 \\
\hline 7 & $-0.000000165+$ i 1.732050808 & 1.732050808 \\
\hline 8 & $-1.732050808+$ i 0.000000010 & 1.732050808 \\
\hline 9 & -0.907604426 - i 11.866568847 & 11.901226911 \\
\hline 10 & -5.959909504 - i 18.342712091 & 19.286669182 \\
\hline 11 & $-17.245203220+\mathrm{i} 14.943053456$ & 22.818673947 \\
\hline 12 & -11.196152781 - i 8.196151933 & 13.875544804 \\
\hline 13 & 0.000000084 - i 15.347547346 & 15.347547346 \\
\hline 14 & 0.000000083 - i 1.732050808 & 1.732050808 \\
\hline 15 & -101.423006915 - i 32.954328677 & 106.642459228 \\
\hline 16 & $-1.224744868+$ i 1.224744875 & 1.732050808 \\
\hline 17 & $-20.645987906+$ i 15.591127116 & 25.871607244 \\
\hline 18 & -40.127945922 - i 30.808776018 & 50.590836361 \\
\hline 19 & 65.239497521 - i 83.819706393 & 106.216454548 \\
\hline 20 & -115.811330427 - i 84.141852139 & 143.150674244 \\
\hline 21 & -53.061367118 - i 135.569663547 & 145.583798394 \\
\hline 22 & $36.010672960+$ i 16.445523443 & 39.588177633 \\
\hline 23 & -40.070955963 - i 2.740927872 & 40.164588848 \\
\hline 24 & 164.290883844 - i 129.064834808 & 208.923972053 \\
\hline 25 & 274.923532195 - i 34.730921473 & 277.108616721 \\
\hline 26 & 0.000000762 - i 277.056941014 & 277.056941014 \\
\hline 27 & $152.288761117+$ i 30.720691710 & 155.356453557 \\
\hline 28 & $-0.000003253+$ i 136.433611353 & 136.433611353 \\
\hline 29 & $-4.270422302+\mathrm{i} 12.674160517$ & 13.374260781 \\
\hline 30 & $485.145409358+$ i 667.745345688 & 825.378649414 \\
\hline 31 & $13.416605095+$ i 0.680413518 & 13.433847358 \\
\hline 32 & $164.960256203+$ i 68.328775084 & 178.551694562 \\
\hline 33 & $-82.729230504+$ i 287.059281883 & 298.742626511 \\
\hline 34 & -538.981501170 - i 268.380890132 & 602.104111256 \\
\hline 35 & $-415.659227492+$ i 629.696787632 & 754.513510650 \\
\hline 36 & $-681.771583909+$ i 223.335013764 & 717.419696551 \\
\hline 37 & 101.630605413 - i 150.367371847 & 181.491395038 \\
\hline 38 & $-59.482939675+$ i 173.267881065 & 183.193828283 \\
\hline 39 & -279.481438371 - i 847.784737546 & 892.663898458 \\
\hline 40 & 347.379313920 - i 1069.123643311 & 1124.143119192 \\
\hline 41 & -941.842709163 - i 512.157840150 & 1072.088308877 \\
\hline 42 & 399.545316356 - i 429.453517605 & 586.572061733 \\
\hline 43 & $390.071778264+$ i 279.072428091 & 479.622155784 \\
\hline 44 & $24.267771316+$ i 37.761389387 & 44.887049949 \\
\hline 45 & $2753.539308039+$ i 289.408610795 & 2768.706568944 \\
\hline 46 & 32.920210846 - i 30.745331135 & 45.044596443 \\
\hline 47 & 536.002877882 - i 206.437637985 & 574.382784800 \\
\hline 48 & $394.055675294+$ i 817.737030229 & 907.729985094 \\
\hline 49 & $-1754.712300120+$ i 400.501606784 & 1799.837990828 \\
\hline 50 & $137.556258644+$ i 2186.393963508 & 2190.716843400 \\
\hline
\end{tabular}




\begin{tabular}{|c|c|c|}
\hline$\bullet$ & $L_{15 / 2}$ & $\bullet$ \\
\hline$k$ & $\overline{I_{k}}$ & $\left|I_{k}\right|$ \\
\hline 3 & $1.000000000+$ i 0.000000000 & 1.000000000 \\
\hline 4 & 0.000000000 - i 1.732050808 & 1.732050808 \\
\hline 5 & $0.000000000+$ i 0.000000000 & 0.000000000 \\
\hline 6 & 3.000000000 - i 3.464101615 & 4.582575695 \\
\hline 7 & 0.000000000 - i 1.732050808 & 1.732050808 \\
\hline 8 & $1.732050808+$ i 0.000000000 & 1.732050808 \\
\hline 9 & 10.730551990 - i 5.147276559 & 11.901226911 \\
\hline 10 & $0.000000000+$ i 0.000000000 & 0.000000000 \\
\hline 11 & $-12.336706536+\mathrm{i} 19.196290072$ & 22.818673947 \\
\hline 12 & $-11.196152423+$ i 8.196152423 & 13.875544804 \\
\hline 13 & 14.350206054 - i 5.442315293 & 15.347547346 \\
\hline 14 & $0.000000000+$ i 1.732050808 & 1.732050808 \\
\hline 15 & $0.000000000+$ i 0.000000000 & 0.000000000 \\
\hline 16 & 1.224744871 - i 1.224744871 & 1.732050808 \\
\hline 17 & $9.345902508+$ i 24.124555285 & 25.871607244 \\
\hline 18 & -6.617211192 - i 50.156208387 & 50.590836361 \\
\hline 19 & 50.553444640 - i 93.414583721 & 106.216454548 \\
\hline 20 & $0.000000000+$ i 0.000000000 & 0.000000000 \\
\hline 21 & $-53.061366041+$ i 135.569663969 & 145.583798394 \\
\hline 22 & $-11.153278505+$ i 37.984578277 & 39.588177633 \\
\hline 23 & -29.353726021 - i 27.414466365 & 40.164588848 \\
\hline 24 & -164.290886665 - i 129.064831217 & 208.923972053 \\
\hline 25 & $0.000000000+$ i 0.000000000 & 0.000000000 \\
\hline 26 & $228.013392388+$ i 157.386281027 & 277.056941014 \\
\hline 27 & $56.698638283+$ i 144.640561664 & 155.356453557 \\
\hline 28 & 0.000000000 - i 136.433611353 & 136.433611353 \\
\hline 29 & $11.816320486+$ i 6.264616638 & 13.374260781 \\
\hline 30 & $0.000000000+$ i 0.000000000 & 0.000000000 \\
\hline 31 & -1.359079795 - i 13.364922631 & 13.433847358 \\
\hline 32 & $164.960256101+$ i 68.328775330 & 178.551694562 \\
\hline 33 & -224.792217910 - i 196.762841162 & 298.742626511 \\
\hline 34 & -110.636340119 - i 591.852144574 & 602.104111256 \\
\hline 35 & $0.000000000+$ i 0.000000000 & 0.000000000 \\
\hline 36 & $147.472006710+\mathrm{i} 702.099015977$ & 717.419696551 \\
\hline 37 & $45.731849880+$ i 175.635202563 & 181.491395038 \\
\hline 38 & -177.588145856 - i 44.971426177 & 183.193828283 \\
\hline 39 & -875.291194999 - i 175.254556482 & 892.663898458 \\
\hline 40 & $0.000000000+$ i 0.000000000 & 0.000000000 \\
\hline 41 & $1052.478012596+$ i 204.116082250 & 1072.088308877 \\
\hline 42 & $399.545318063+$ i 429.453516017 & 586.572061733 \\
\hline 43 & -171.335849479 - i 447.974819607 & 479.622155784 \\
\hline 44 & $44.430164502+$ i 6.388093254 & 44.887049949 \\
\hline 45 & $0.000000000+$ i 0.000000000 & 0.000000000 \\
\hline 46 & -17.945816315 - i 41.315412930 & 45.044596443 \\
\hline 47 & $571.498128245+\mathrm{i} 57.493242102$ & 574.382784800 \\
\hline 48 & -817.737034535 - i 394.055666358 & 907.729985094 \\
\hline 49 & -780.920437266 - i 1621.597997004 & 1799.837990828 \\
\hline 50 & $0.000000000+$ i 0.000000000 & 0.000000000 \\
\hline
\end{tabular}




\begin{tabular}{|c|c|c|}
\hline$\bullet$ & $L_{15 / 4}$ & $\bullet$ \\
\hline$k$ & $\overline{I_{k}}$ & $\left|I_{k}\right|$ \\
\hline 3 & $1.000000000+$ i 0.000000000 & 1.000000000 \\
\hline 4 & $0.000000000+$ i 1.732050808 & 1.732050808 \\
\hline 5 & -1.018073921 - i 3.133309346 & 3.294556414 \\
\hline 6 & $3.000000000+$ i 3.464101615 & 4.582575695 \\
\hline 7 & -0.751508681 - i 1.560523855 & 1.732050808 \\
\hline 8 & $-1.732050808+$ i 0.000000000 & 1.732050808 \\
\hline 9 & $10.730551990+$ i 5.147276559 & 11.901226911 \\
\hline 10 & -15.603243133 - i 11.336419711 & 19.286669182 \\
\hline 11 & $17.245203123+$ i 14.943053568 & 22.818673947 \\
\hline 12 & -11.196152423 - i 8.196152423 & 13.875544804 \\
\hline 13 & $3.672908488+$ i 14.901575513 & 15.347547346 \\
\hline 14 & $-1.688624678+$ i 0.385417563 & 1.732050808 \\
\hline 15 & $0.000000000+$ i 0.000000000 & 0.000000000 \\
\hline 16 & $-1.224744871+$ i 1.224744871 & 1.732050808 \\
\hline 17 & -17.429589094 - i 19.119348456 & 25.871607244 \\
\hline 18 & $-6.617211192+$ i 50.156208387 & 50.590836361 \\
\hline 19 & 0.000000000 - i 106.216454548 & 106.216454548 \\
\hline 20 & $-44.235991098+$ i 136.144381552 & 143.150674244 \\
\hline 21 & 72.909410760 - i 126.011349400 & 145.583798394 \\
\hline 22 & $-36.010673013+$ i 16.445523326 & 39.588177633 \\
\hline 23 & $-10.836276386+$ i 38.675176941 & 40.164588848 \\
\hline 24 & 164.290886665 - i 129.064831217 & 208.923972053 \\
\hline 25 & $-257.649075864+$ i 102.010485575 & 277.108616721 \\
\hline 26 & 275.036883975 - i 33.395523912 & 277.056941014 \\
\hline 27 & $-153.611719961+$ i 23.217819719 & 155.356453557 \\
\hline 28 & $106.668092622+$ i 85.064965309 & 136.433611353 \\
\hline 29 & $-9.197471902+$ i 9.709653035 & 13.374260781 \\
\hline 30 & $0.000000000+$ i 0.000000000 & 0.000000000 \\
\hline 31 & $-4.021598499+$ i 12.817761129 & 13.433847358 \\
\hline 32 & -164.960256101 - i 68.328775330 & 178.551694562 \\
\hline 33 & $85.599713692+$ i 286.216431937 & 298.742626511 \\
\hline 34 & -217.505092368 - i 561.445362956 & 602.104111256 \\
\hline 35 & $33.851120653+$ i 753.753765751 & 754.513510650 \\
\hline 36 & 147.472006710 - i 702.099015977 & 717.419696551 \\
\hline 37 & $-136.240563896+$ i 119.906777214 & 181.491395038 \\
\hline 38 & $0.000000000+$ i 183.193828283 & 183.193828283 \\
\hline 39 & 538.949599873 - i 711.605343156 & 892.663898458 \\
\hline 40 & $-909.450887536+$ i 660.754746927 & 1124.143119192 \\
\hline 41 & 1008.985242455 - i 362.383943545 & 1072.088308877 \\
\hline 42 & $-546.310790198+$ i 213.568031595 & 586.572061733 \\
\hline 43 & $426.548198677+$ i 219.303548819 & 479.622155784 \\
\hline 44 & $-24.267771377+$ i 37.761389348 & 44.887049949 \\
\hline 45 & $0.000000000+$ i 0.000000000 & 0.000000000 \\
\hline 46 & $-6.133571758+$ i 44.625048641 & 45.044596443 \\
\hline 47 & -558.735830232 - i 133.153503483 & 574.382784800 \\
\hline 48 & $394.055666358+$ i 817.737034536 & 907.729985094 \\
\hline 49 & -883.212092863 - i 1568.232505801 & 1799.837990828 \\
\hline 50 & $410.499402001+$ i 2151.913225229 & 2190.716843400 \\
\hline
\end{tabular}

